\title{
JNFORIMIACJÓN
}

\section{Incidencia del modo de explotación del olivo sobre la renta neta del olivicultor. Estrategias para el cultivo extensivo en el contexto de la posible ausencia de subvenciones}

\author{
Por Juan Vilar Hernández, ${ }^{1,2}$ María del Mar Velasco Gámez ${ }^{1}$ y Raquel Puentes Poyatos ${ }^{1}$ \\ ${ }^{1}$ Departamento de Administración de Empresas, Contabilidad y Sociología. Universidad de Jaén. \\ Campus de Las Lagunillas s/n, 23.071 Jaén-España \\ ${ }^{2}$ GEA Westfalia Separator Ibérica, S.A. Centro de Desarrollo y Competencia para Aceite de Oliva \\ P.I. Los Cerros. Cerámica, naves 4, 5 y 6, 23400 Úbeda - España \\ ( ${ }^{*}$ Autor para correspondencia: Juan.Vilar@geagroup.com )
}

\section{RESUMEN}

Incidencia del modo de explotación del olivo sobre la renta neta del olivicultor. Estrategias para el cultivo extensivo en el contexto de la posible ausencia de subvenciones.

El presente trabajo trata de analizar la competitividad, en términos de rentabilidad, de las explotaciones de olivar tradicional en relación con las nuevas modalidades de cultivo de mayor densidad de plantación, a la vez que se enuncian una serie de alternativas, que podrían ayudar a consolidar su futuro, vía incremento de rentabilidad. Los resultados muestran como las explotaciones tradicionales en ausencia de ayudas son menos rentables que las intensivas y superintensivas, por lo que sería conveniente que adoptaran algunas de las estratégicas de mejora de la competitividad que aquí se enuncian, consistentes principalmente en la innovación incremental, la agrupación, o la explotación conjunta.

PALABRAS CLAVE: Aceite de oliva - Competitividad Olivar tradicional - Rentabilidad.

\section{SUMMARY}

Influence of the olive exploitation system on the net profit of the producer. Strategies for extensive olive fields in the context of the absence of subsidies.

This article compares the competiveness and profit margins of different cultivation systems and analyzes these circumstances for this agricultural activity and at the same time gives some different alternatives in order to help to improve the net profit from traditional olive tree cultivation. These different strategies are always based on field concentration, work force and innovation activity.

KEY-WORDS: Competitiveness - Olive oil - Profitability - Traditional olive growing.

\section{INTRODUCCIÓN}

La agricultura de la Unión Europea, donde se integran los principales países productores de aceite de oliva a nivel mundial, con mayoría de explotaciones extensivas, ha sido objeto tradicionalmente de una elevada protección estatal (Tamames y Rueda, 2005; Lamo de Espinosa, 2008).

Con las sucesivas modificaciones experimentadas por la PAC tendentes a reducir los mecanismos de apoyo que tradicionalmente venía aplicando a los agricultores, se han articulado las ayudas directas parcial y totalmente desconectadas de la producción, complementadas con medidas estructurales, agroambientales y de control efectivo de la producción, con lo que se ha perseguido incrementar la eficiencia y la competitividad de la agricultura europea (Massot, 2005; Tió, 2007). Se apuesta por la sostenibilidad y multifuncionalidad agraria (Atance y Tió, 2000; Atance et al., 2001; Reig, 2002, 2007), fundamentada en la protección medioambiental, la seguridad alimentaria, las políticas de calidad, así como por la cohesión económica y social (Massot, 2005; Puentes y Velasco, 2009).

Las explotaciones que integran el sector del olivar europeo, son las que tradicionalmente han venido percibiendo las ayudas comunitarias, y por tanto, las que han quedado incorporadas al Régimen de Pago Único, y sujetas a los principios de una PAC reformada. En estas explotaciones, se emplea un modo de cultivo tradicional y es propio de los países con tradición olivarera como España (primer país productor mundial de aceite de oliva), Italia o Grecia.

La importancia de este tipo de explotaciones viene justificada por el hecho de que de un total de 11 millones de hectáreas (en adelante ha) dedicadas al cultivo de olivar en el mundo, 8 millones, es decir, el $73 \%$ se encuentran pobladas por olivar marginal y tradicional, ubicadas principalmente en la cuenca mediterránea donde tiene su origen y donde más arraigado se encuentra este tipo de cultivo (MARM, 2009). No obstante, hoy en día el cultivo de esta planta ha llegado a más de 30 países de los 5 continentes, plantándose incluso en territorios sin ninguna tradición olivarera, un claro ejemplo sería China, alcanzando gran importancia el territorio dedicado a dichas explotaciones. 
Ante estas realidades, se hace necesaria la implantación de una estrategia competitiva para las fincas tradicionales de olivar europeo, reorientando sus actuaciones, su explotación y conducta.

Ahora se les exige básicamente eficiencia, es decir una relación óptima entre inputs y outputs, lo que se consigue con los nuevos sistemas de cultivo con plantaciones de mayor densidad, hacia los que han ido evolucionando las explotaciones tradicionales, conocidos como "la nueva olivicultura" (Barranco, 2007a; Tous et al., 2007; Pastor et al., 2007) fácilmente mecanizables, que conllevan a un incremento de la rentabilidad del olivicultor vía reducción de costes de producción, básicamente el de recolección, resultando por tanto más competitivas que las anteriores, que se están implantando principalmente en los países de nueva incorporación al cultivo del olivo, sin ningún arraigo o tradición.

Tomando como referencia lo anteriormente argumentado, el objetivo de esta investigación es presentar la competitividad de las explotaciones tradicionales de olivar en relación con las nuevas modalidades de cultivo de mayor densidad de plantación, y proponer actuaciones de mejora competitiva. El método seguido ha consistido en la realización de un trabajo de campo en el que se compara la competitividad de una explotación tradicional, con otra intensiva y superintensiva. Para alcanzar el objetivo propuesto el trabajo se ha estructurado como sigue. Tras esta introducción, se dedica un segundo epígrafe a presentar la relevancia del sector de elaboración de aceite de oliva a nivel mundial, efectuando una comparativa de la competitividad, en base a la rentabilidad, de los distintos modos de explotación. En el tercer epígrafe y a la vista de los resultados obtenidos, se muestran las estrategias de futuro para la mejora de la competitividad del cultivo tradicional.

\section{CONSIDERACIONES SOBRE EL SECTOR OLIVARERO: ANÁLISIS COMPARATIVO DE LOS DISTINTOS MODOS DE EXPLOTACIÓN}

Actualmente se producen en el mundo más de 10 tipos distintos de grasas vegetales (Tabla 1), suponiendo por campaña (2008/2009) la producción total del orden de 140 millones de toneladas, de éstas las de mayor proporción son las de: palma $(25 \%)$, soja $(25 \%)$, colza (12\%) y girasol $(7 \%)$, conformando de modo conjunto más del $68 \%$ sobre el total, casi 100 millones de toneladas. Si a dichos aceites vegetales se adiciona la producción de grasas, sebos y mantecas animales (26 millones de toneladas), la cuantificación total obtenida es de 139,5 millones de toneladas, por campaña, tratándose de un mercado en equilibrio, pues la totalidad de dicha producción es absorbida. La producción de aceite de oliva supuso para dicha campaña el $2,07 \%$ del total de la producción de aceites y grasas en el mundo.

La producción anual media de aceite de oliva (tabla 2) ha experimentado en los últimos cuatro años un ascenso anual de 3,1 puntos porcentuales llegando a alcanzar los 2,8 millones de $t$, al igual que el consumo medio, que lo hace en torno al $3,4 \%$, rebasando los 2,8 millones de $t$ dando lugar a un stock anual medio de 0,18 millones de t. Los mismos datos referidos a la aceituna de mesa se recogen en la Tabla 3.

A nivel mundial, la importancia del sector oleícola distribuido por continentes se presenta en la Tabla 4 y Figura 1, y como se puede apreciar, Europa, ocupa la primera posición en todos y cada uno de los aspectos señalados, seguida a gran distancia por el continente Africano.

Otros datos que ponen de manifiesto su importancia económica son que la cifra de negocios que presenta el sector ha llegado a alcanzar cantidades que oscilan entre 8.500 y 10.600 millones de euros por ejercicio. La superficie dedicada al cultivo, es de entre 150.000 a 300.000 ha por campaña, lo que se traduce en 35 ó 45 millones de plantones $u$ olivos nuevos por año.

Por último, se ha de indicar que del total de 11 millones de ha dedicadas al cultivo de olivar desde el punto de vista mundial, 8 millones de ha (73\%) se encuentran pobladas de olivar tradicional y marginal; 2,9 millones de ha (26\%) se destinan a olivar intensivo, mientras que solo 90.000 ha (casi el 1\%) se utilizan para el cultivo superintensivo de olivar.

\subsection{Modos de explotación del olivar. Comparativa}

Aun cuando existen autores que establecen solo 3 distintos sistemas de cultivo y explotación del olivo, a continuación se describen, los que a nuestro juicio existen:

\section{Olivar marginal}

El Olivar marginal o de bajo rendimiento, es aquel que presenta rentabilidades nulas o muy bajas, por consiguiente es susceptible de abandono por habitualmente ser improbable su adecuación a otro cultivo; engloba el de montaña en pendientes

Tabla 1

Producción de aceites y grasas en el mundo 2008/2009

\begin{tabular}{lccccccccccc}
\hline Aceite & Soja & Algodón & Mani & Girasol & Colza & Palma & Maíz & Oliva & Coco & Animal & Total \\
\hline Cantidad & 34,3 & 4,9 & 4,6 & 10,0 & 16,9 & 34,9 & 2,1 & 2,9 & 3,1 & 25,8 & $\mathbf{1 3 9 , 5}$ \\
\hline
\end{tabular}

*Datos en Millones t. Fuente: Elaboración propia a partir de datos ASAGA (2009). 
Tabla 2

Producción, consumo, exportaciones, importaciones y stock anual mundial de aceite de oliva (1997-2009)

\begin{tabular}{|c|c|c|}
\hline Campañas & $\begin{array}{c}1997 / 98- \\
2002 / 03\end{array}$ & $\begin{array}{c}2003 / 04- \\
2008 / 2009\end{array}$ \\
\hline Producción & $2.521,5$ & $2.837,6$ \\
\hline Consumo & $2.517,1$ & $2.819,3$ \\
\hline Importaciones & 455,5 & 626,2 \\
\hline Exportaciones & 482,4 & 654,6 \\
\hline Stock & 4,4 & 18,3 \\
\hline
\end{tabular}

* Miles de t. Fuente: Consejo Oleícola Internacional (2009).

Tabla 3

Producción, consumo, exportaciones, importaciones y stock anual mundial de aceituna de mesa (1997-2009)

\begin{tabular}{lcc}
\hline Campañas & $\begin{array}{c}\mathbf{1 9 9 7 / 9 8 -} \\
\mathbf{2 0 0 2 / 0 3}\end{array}$ & \begin{tabular}{c}
$\mathbf{2 0 0 3 / 0 4} \mathbf{2 0 0 8 / 2 0 0 9}$ \\
\hline Producción
\end{tabular} 1.429,1 $^{1.910,2}$ \\
Consumo & $1.258,6$ & $2.036,6$ \\
Importaciones & 357,5 & $517,, 2$ \\
Exportaciones & 356,8 & 545,5 \\
Stock & 170,5 & $-126,40$ \\
\hline
\end{tabular}

${ }^{*}$ Miles de t. Fuente: Consejo Oleícola Internacional (2009).

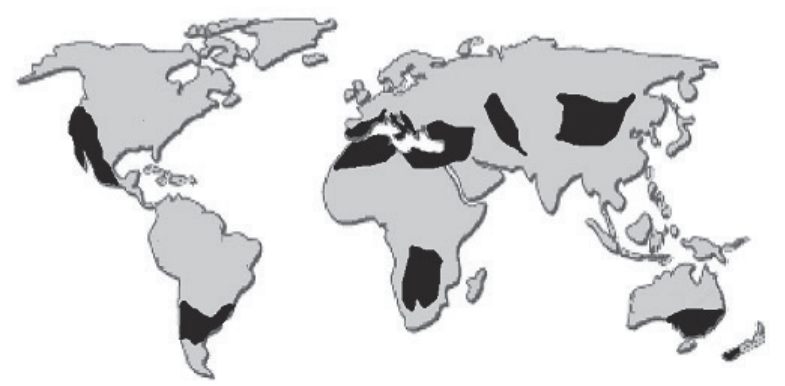

Figura 1.

Distribución geográfica a nivel mundial del cultivo del olivo. Fuente: Elaboración propia a partir del Consejo Oleícola Internacional (2009)

pronunciadas, altitud superior a 1.000 metros (y la combinación de ambos factores) y aquel que por circunstancias orográficas o climáticas hacen que resulte difícilmente mecanizable. Se trata de explotaciones dispersas que en ocasiones no superan la hectárea, con plantas de más de 50 - 60 años, y habitualmente entre 3 y 5 pies (Arriaza et al., 2002; Mulero, 2003; AEMO, 2003; Vilar y Velasco, 2007):

\section{Olivar Tradicional o extensivo}

Plantados en tierras de secano, aunque a veces también de regadío, generalmente superan los 100 años, con marcos de plantación muy amplios entre
70 - 100 olivos por ha (Navarro y Parra, 1997), dispuestos en cuadrículas de $10 \times 10,11 \times 11,12 \times$ 12, $15 \times 15$, (marco real y tresbolillo), e incluso $24 \times$ 24 , como es el caso de los cultivos de la zona de Sfax (Túnez), de grandes dimensiones, con 2 o 3 pies, ello hace difícil su mecanización y por tanto antieconómico (Barranco, 2007a).

Este tipo de cultivo es el habitual en zonas de gran tradición olivarera, provincias como Jaén, Córdoba, Granada o Sevilla y en a países como España, Italia, Grecia, Turquía, Siria, Túnez, etc. igualmente se ven afectados por una gran dispersión tanto geográfica como en cuanto a propiedad de las explotaciones. La producción media ronda de 1.000 a $4.000 \mathrm{Kg}$. por ha, en secano y de 4.000 a 8.000 en regadío, el mayor nivel de ingresos proviene de las subvenciones.

En cuanto, a la competitividad de este tipo de explotaciones, a continuación, se procede a analizar la rentabilidad de una explotación tradicional, que ha percibido la subvención de la PAC en la campaña 2007/2008. Como se puede apreciar, en la tabla 5, los costes de recolección suponen el $42,88 \%$ de los gatos totales, alcanzando el $48,07 \%$ de la renta neta del olivicultor, mientras que por otro lado, la subvención a la producción oscila en torno al $41,22 \%$ de la renta neta y representa el $19,43 \%$ del total de ingresos, cubriendo con esta subvención el $85,76 \%$ de los costes de recolección, los más elevados. En términos generales, se podría asegurar, que a través de la subvención a la producción, el olivicultor, cubre entre el 80 y $100 \%$ de los costes de recolección, circunstancia esta que hace apta la renta neta del productor vía compensación, entre el resto de ingresos y gastos de explotación.

Como se ha dicho, se produce un incremento de la competitividad de tal sistema de explotación, vía compensación de costes de recolección, a través de los ingresos procedentes de la subvención a la producción. La razón básica de dichos costes tan elevados y la baja rentabilidad provienen de la escasa posibilidad de mecanización, así como, de la proporcionalmente inferior masa vegetal productiva expuesta a radiación solar, comparando con otros sistemas de explotación. Del mismo modo tal sistema de cultivo, se ve afectado por un elevado nivel de dispersión, lo que encarece los costes de transporte y dificulta la adquisición o disponibilidad de maquinaria cuando la propiedad está muy atomizada.

\section{Olivar Intensivo}

Un cultivo intensivo se caracteriza por aunar del orden de entre 200 y 600 olivos por ha, así como buenas condiciones, orográficas y climáticas, todos los olivos han sido tutorados y tienen un solo pie, lo que aumenta las posibilidades de mecanización, a la vez que incrementa la masa vegetal expuesta al sol, ambas circunstancias potencian la rentabilidad y reducen los costes; la producción oscila de 5.000 a $6.000 \mathrm{Kg}$. por ha en secano, y de 8.000 a $12.000 \mathrm{Kg}$. por ha en riego, los marcos de plantación son de 
Tabla 4

Distribución continental de magnitudes oleícolas

\begin{tabular}{ccccc}
\hline Continente & $\begin{array}{c}\text { Olivos } \\
\text { (unidades) }\end{array}$ & $\begin{array}{c}\text { Superficie } \\
\text { (hectáreas) }\end{array}$ & $\begin{array}{c}\text { Producción } \\
(\mathbf{t})\end{array}$ & $\begin{array}{c}\text { Consumo } \\
(\mathbf{t})\end{array}$ \\
\hline África & 252.500 .000 & 2.816 .900 & 305.700 & 180.350 \\
América & 37.800 .000 & 190.350 & 30.600 & 306.980 \\
Asia & 138.300 .000 & 986.400 & 206.610 & 195.610 \\
Europa & 964.400 .000 & 6.508 .100 & 2.310 .600 & 1.893 .900 \\
Oceanía & 7.000 .000 & 40.000 & 13.000 & 45.000 \\
\hline
\end{tabular}

Fuente: Adaptado y completado de Consejo Oleícola Internacional (2009).

Tabla 5

Análisis económico de actividad para cultivo tradicional

\begin{tabular}{|c|c|c|}
\hline Producción por hectárea & $\begin{array}{l}\text { Gastos } \\
\text { (euros) }\end{array}$ & $\begin{array}{c}\text { Ingresos } \\
\text { (euros) }\end{array}$ \\
\hline \multicolumn{3}{|l|}{ kg /olivo $(54,05)$} \\
\hline Ingresos (Kg. 0,53 euros) & & $3.171,57$ \\
\hline Subvención a la produc. (0,13 euros por Kg.) & & 765,18 \\
\hline Poda y eliminación de leña (Bianual) & 153,00 & \\
\hline Arijas (arado, rastreado, etc.) & 154,00 & \\
\hline Abonado / estercolado & 186,00 & \\
\hline Tratamientos (repilo, prays y mosca) & 109,00 & \\
\hline Despestugo / desvareto & 87,00 & \\
\hline Recolección & 892,21 & \\
\hline Transporte de tajo a almazara & 58,00 & \\
\hline Extracción (euros / Kg.) & 0,09 & \\
\hline Total & $2.080,66$ & $3.936,75$ \\
\hline Renta Neta & \multicolumn{2}{|c|}{$1.856,09$} \\
\hline Valor inmovilizado de mercado (euros) & \multicolumn{2}{|c|}{$36.060,72$} \\
\hline Margen neto de Explotación (\%) & \multicolumn{2}{|c|}{$5,15 \%$} \\
\hline
\end{tabular}

Fuente: Elaboración propia a partir de datos de la explotación Tronco Seco ubicada en Chilluévar (Jaén-España) (campaña 2007/08).

$5 \times 8,6$ × 8, 7 × 4 etc., (Humanes et al., 1998; Barranco, 2007a).

Por sus características, en el análisis de la rentabilidad de una explotación de cultivo intensivo, se advierte (Tabla 6) que los costes de recolección solo suponen el $8,6 \%$ de la renta neta, del mismo modo que son íntegramente cubiertos por la subvención a la explotación, suponiendo aquellos el 32\% de esta. Comparativamente tal cultivo, es más efectivo que el anterior, por razones de competitividad (los costes son más bajos, en torno al 50\%, especialmente el de recolección) y productividad (la producción es superior en intervalos de 20 a $40 \%$ ). No obstante, se ha de señalar, que este análisis se ha llevado a cabo en una explotación intensiva, cuya producción de referencia es anterior a mayo de 1998, fecha límite para tener derecho a la subvención a la producción concedida vía PAC.

A continuación (Tabla 7) se expone el mismo análisis, pero para una explotación intensiva que no percibe subvención, situada en la comarca del Alentejo Portugues.

Como se puede observar, y en comparación con el caso anterior, se produce un descenso de los ingresos totales en un 23,09\%, por motivo de la no percepción de la subvención a la producción, del mismo modo, que se produce un retroceso en los costes globales, vía reducción del coste de recursos humanos. Otra circunstancia a destacar, sería el incremento del valor porcentual de margen bruto, debido al descenso del valor de mercado de la tierra en la zona portuguesa de referencia.

A la vista de los resultados, se podría aseverar, que el olivar intensivo (en este caso no al $100 \%$ de su nivel productivo), resulta competitivo por si mismo, sin necesidad de subvención a la producción, o de manera más concreta, sin percepción de ingresos diferentes a los de explotación, siempre y cuando se mantengan las actuales cotas para el precio del aceite. 
Tabla 6

Análisis económico de actividad para cultivo intensivo

\begin{tabular}{|c|c|c|}
\hline Producción por hectárea & $\begin{array}{l}\text { Gastos } \\
\text { (euros) }\end{array}$ & $\begin{array}{c}\text { Ingresos } \\
\text { (euros) }\end{array}$ \\
\hline \multicolumn{3}{|l|}{ kg /olivo $(22,60)$} \\
\hline Ingresos (Kg. 0,52 euros) & & $5.876,00$ \\
\hline Subvención a la produc. (Kg. 0,13 euros) & & $1.426,20$ \\
\hline Poda y eliminación de leña (Bianual) & 123,56 & \\
\hline Arijas (arado, rastreado, etc.) & 93,47 & \\
\hline Abonado / estercolado/ regado & 65,12 & \\
\hline Tratamientos (repilo, prays y mosca) & 96,78 & \\
\hline Despestugo / desvareto & 20,38 & \\
\hline Recolección & 456,03 & \\
\hline Transporte de tajo a almazara & 135,82 & \\
\hline Extracción (euros / Kg.) & 0,09 & \\
\hline Total & $2.008,16$ & $7,302,20$ \\
\hline Renta Neta & \multicolumn{2}{|c|}{$5.294,04$} \\
\hline Valor inmovilizado de mercado (euros) & \multicolumn{2}{|c|}{$72.500,00$} \\
\hline Margen neto de Explotación (\%) & \multicolumn{2}{|c|}{$7,30 \%$} \\
\hline
\end{tabular}

Fuente: Elaboración propia a partir de datos de la explotación La Dorada ubicada en

Término Municipal de Jaén (España) (campaña 2007/08).

Tabla 7

Análisis económico de actividad para cultivo intensivo

\begin{tabular}{|c|c|c|}
\hline Producción por hectárea & $\begin{array}{l}\text { Gastos } \\
\text { (euros) }\end{array}$ & $\begin{array}{c}\text { Ingresos } \\
\text { (euros) }\end{array}$ \\
\hline \multicolumn{3}{|l|}{$\mathrm{kg}$ /olivo (25.00) } \\
\hline Ingresos (Kg. 0,52 euros) & & 5.616 \\
\hline Subvención a la produc. (Kg. 0,00 euros) & & 0 \\
\hline Recolección & 408,00 & \\
\hline Otros gastos de explotación & 870,00 & \\
\hline Extracción (euros / Kg.) & 0,06 & \\
\hline Total & $1.890,00$ & $5.616,00$ \\
\hline Renta Neta & \multicolumn{2}{|c|}{$3.726,00$} \\
\hline Valor inmovilizado de mercado (euros) & \multicolumn{2}{|c|}{$30.000,00$} \\
\hline Margen neto de Explotación (\%) & \multicolumn{2}{|c|}{$12,00 \%$} \\
\hline
\end{tabular}

\section{Olivar superintensivo o de alta densidad}

Es propio de recientes plantaciones en los nuevos productores internacionales (Argentina, Australia, China, Chile, etc.) (Vilar et al., 2005; Vilar, 2006).

Resulta imprescindible el riego por goteo, un perfecto conocimiento olivarero, unos elevados costes de iniciación por guío o tutoría de plantas, mecanización, inmovilizado, etc., sin embargo, durante los primeros años, el rendimiento es inigualable, considerado este modelo por tanto más adecuado para grandes superficies, el área mínima de optimización debe superar las 15 ha. (León et al., 2006; Tous et al., 2007; Barranco, 2007b).
Los árboles se disponen de norte a sur y con un marco habitual de $4 \times 2 ; 4,5 \times 2,5 ; 4 \times 1,5$, etc. del mismo modo que se ve afectado por la orografía del terreno y orientación, lo hace por el diseño y tamaño de los elementos recolectores, entre otras cosas. Las producciones obtenidas oscilan entre 10.000 y $15.000 \mathrm{Kg}$. por ha. (Pastor et al., 2005).

En la Tabla 8 se presenta el análisis económico de actividad para cultivo superintensivo donde se aprecia que este sistema de explotación presenta el mayor margen de explotación comparativo, debido fundamentalmente a 3 factores: el incremento de productividad, el decremento de costes vía reducción de gastos de recolección y el descenso del valor de inmovilizado. 
Tabla 8

Análisis económico de actividad para cultivo superintensivo

\begin{tabular}{lcc}
\hline Producción por hectárea & $\begin{array}{c}\text { Gastos } \\
\text { (euros) }\end{array}$ & $\begin{array}{c}\text { Ingresos } \\
\text { (euros) }\end{array}$ \\
\hline kg /olivo (9,12) & $7,098,00$ \\
Ingresos (Kg. 0,59 euros) & & \\
Subvención a la produc. (Kg. 0,21 euros) & 140,87 \\
Poda y eliminación de leña (Bianual) & 102,74 \\
Arijas (arado, rastreado, etc.) & 94,80 \\
Abonado / estercolado/ regado & 178,09 \\
Tratamientos (repilo, prays y mosca) & 37,80 \\
Despestugo / desvareto & 360,00 \\
Recolección & 158,08 \\
Transporte de tajo a almazara & 0,10 \\
Extracción (euros / Kg.) & $2.272,38$ \\
Total & \multicolumn{2}{c}{$4.825,62$} \\
Renta Neta & \multicolumn{2}{c}{47.000} \\
Valor inmovilizado de mercado (euros) & \multicolumn{2}{c}{$8,87 \%$} \\
Margen neto de Explotación (\%) &
\end{tabular}

Fuente: Elaboración propia a partir de datos de la explotación La Loma ubicada en Término Municipal Écija (España) (campaña 2007/08).

\section{RETOS Y ESTRATEGIAS DE FUTURO PARA EL CULTIVO TRADICIONAL O EXTENSIVO}

Hasta ahora se ha puesto de manifiesto, en las distintas tablas, que las explotaciones tradicionales son menos competitivas, en términos de rentabilidad, que las otras modalidades de explotación, intensiva y superintensiva. Si a esto se une la escasez cada vez más acusada de mano de obra para la realización de las tareas agrícolas propias de esta actividad; los elevados costes de producción; la búsqueda de una mejora continua de la calidad de los aceites que los hagan ser más competitivos; la orientación a la constante reducción de costes que hagan económicamente rentables nuestros olivares en un horizonte posterior a 2013 o la previsión de que la producción global de aceite de oliva para 2017 alcanzará valores en torno a 4,0 millones de t (COI, 2009), se pueden considerar como motivos suficientes que justifiquen el inicio de una auténtica revolución en cuanto a las formas de cultivo del olivar.

A dichos matices se ha de adicionar que de las 2.513.419 ha de olivar que actualmente se cultivan en España, 815.000 (32,27\%) se destinaron a tal cultivo con posterioridad a mayo de 1998 (Ministerio de Medio Ambiente, Medio Rural y Marino, 2009), y por consiguiente no perciben ningún tipo de ingreso distinto al de explotación, materializado mediante subvenciones a la producción, con la consiguiente reducción de ingresos que ello supone, afectando negativamente al margen neto de explotación del olivicultor.

Es por todo ello, además que la actividad oleícola supone el $0,6 \%$ del PIB nacional, y es considerada como sector estratégico especialmente en comunidades como Andalucía, y fundamentalmente en provincias como Jaén, Córdoba, Málaga o Sevilla, donde la existencia mayoritaria de explotaciones tradicionales hace necesaria una serie de medidas o estrategias que garanticen o perpetúen su futuro. Para ello, se establecen a continuación cinco puntos básicos en los que basar dicha estrategia de continuidad y diferenciación con respecto a otros productores: concentración de oferta, promoción, competitividad, calidad y seguridad agroalimentaria.

\subsection{Concentración de oferta}

En la actualidad en nuestro país se consumen del orden de 528 millones de litros de aceite de oliva, producidos por 1.725 almazaras, con escasa orientación al mercado y que, por tanto, de forma obligada y urgente, han de comenzar un proceso de concentración de oferta siguiendo los pasos de los 10 mayores grupos envasadores españoles, que desde mediados de 2002 emprendieron un proceso de integración, reduciendo el abanico de operadores en más del 60\% (pasando de 25 a 10); circunstancia ésta que les ha permitido, ejercer una posición de poder a la hora de determinar el precio de aceite de oliva en origen.

\subsection{Promoción}

La previsión de incremento de la producción, para los próximos 7 años es del orden del 38\%, situándose en 4 millones de t para el 2017 según el Consejo Oleícola Internacional. Sin embargo, el consumo está 
sujeto a una serie de variables, económicas sociales y políticas como son el precio, pautas de consumo, preferencias de los consumidores, etc., que lo hacen imprevisible.

Por ello, la promoción es considerada una estrategia de tal importancia para el sector tradicional, que de producirse un descenso, o contención del consumo, la presión de la oferta sobre la demanda, generaría una caída de precios asumibles únicamente por aceites más competitivos, procedentes de cultivos intensivos y superintensivos (COI, 2000).

Por tanto, la promoción del consumo de aceite de oliva no solo en economías emergentes, sino, en el resto del mundo es especialmente vital e importante, con objeto de perpetuar y asegurar el futuro del sector tradicional de producción de aceite de oliva, considerado un factor estratégico de especial importancia potenciar y tener en cuenta de forma obligada no solo en los años próximos, sino desde la actualidad.

\subsection{Calidad}

La calidad aparece como condición necesaria para lograr el éxito en el mercado agroalimentario. La mejora en las cotas de calidad alcanzados por los aceites españoles en los últimos quince años los posicionan como uno de los de mayor rango a nivel internacional, incrementando de manera gradual la composición y proporción de aceite de oliva virgen extra, entre la totalidad de la producción global. (Vilar et al., 2009a).

\subsection{Seguridad agroalimentaria}

En la actualidad la cota de seguridad agroalimentaria, de nuestros aceites se considera adecuada (Alba, 2009) y superior a la existente en otros países igualmente productores de aceite de oliva, principalmente ubicados en Asia o África, al encontrarse ampliamente cubierto por la legislación aplicable de manera obligatoria en el marco UE.

\subsection{Competitividad}

Como se ha dicho con anterioridad, el $32,27 \%$ del olivar plantado en nuestro país no percibe subvención, con lo cual dicha actividad, es económicamente rentable, sin necesidad de ingresos ajenos a la explotación. El liderazgo en costes es una estrategia competitiva (Porter, 2003), que no solo se encuentra constatada para la actividad que nos ocupa, y que por consiguiente puede y debe ser utilizada con éxito, sino que, se trata de la más importante, en la que se debe incidir con mayor intensidad al objeto de mantener, asegurar y defender el futuro del olivar tradicional o extensivo (Leone, 2000).

No obstante se ha de tener en cuenta que alguna de las razones que justifican esta falta de competitividad motivada por los altos costes se en- cuentran en la dimensión reducida de la mayoría de las explotaciones, condiciones climáticas más desfavorecidas, cultivos en zonas que presentan condiciones orográficas inadecuadas, excesiva atomización y dispersión,..., y que en caso de desaparecer las medidas protectoras dejarán de ser explotadas, como fincas de olivar, por no resultar posible su readecuación o adaptación .

Ante tal situación, dicha estrategia puede ser adoptada desde dos ópticas o puntos de vista, según sea aplicada de modo radical, o gradual.

\section{Orientación radical}

Ésta consistiría en renunciar en la actualidad a la ayuda a la producción, y de modo inmediato reordenar o transformar el olivar tradicional en olivar intensivo o superintensivo, ello traería consigo la no percepción de renta en el periodo de entrada en producción del nuevo olivar (aproximadamente 3 ó 4 años), así como, los posteriores años de entrada gradual en plena producción, de este modo, de manera inmediata el olivicultor garantiza las cotas de renta neta anteriormente abordadas para dicho tipo de cultivo o explotación, orientándose hacia un liderazgo en costes, y por consiguiente, alcanzando el nivel adecuado de competitividad (Canals, 1991).

No obstante dicha elección cuenta con una serie de limitaciones, destacando el tamaño de las explotaciones, excesivamente dispersas, y las características orográficas del terreno. En este sentido, para este sistema de cultivo se requiere un área superior a 15 ha (Hidalgo et al., 2006; Abos et al., 2007), y actualmente como se puede apreciar en la Tabla 9, en España el 79,02\% del total de las explotaciones no alcanza dicha superficie mínima.

En la Tabla 10 se puede apreciar la distribución de explotaciones, superficies, tamaños, etc. a nivel mundial, destacando los contextos geográficos con mayor número de explotaciones de olivar como la UE, dentro de ella como país España, como comunidad autónoma Andalucía y como provincia es importante resaltar Jaén, como la primera productora de aceite de oliva con una superficie de olivar de 576.840 ha, ocupadas por 69 millones de olivos distribuidas en 131.000 explotaciones.

A las explotaciones tradicionales sujetas a estas limitaciones les resulta inalcanzable el mínimo de optimización, no consiguiendo todos los efectos competitivamente beneficiosos de una posible transformación, aunque si los aspectos negativos, ante el coste por tutores, mayores amortizaciones, adecuación de maquinaria y no percepción de rentas en periodo transitorio, entre otros.

\section{Orientación gradual}

La orientación gradual consiste en una serie de medidas basadas en el aumento de la coordinación y cooperación, y en una mayor profesionalización del sector (García, 2001) que contribuyen de modo paulatino a la reducción de costes y por consi- 
Tabla 9

Distribución de explotaciones genéricas de cultivo por tamaño (España)

\begin{tabular}{lcccccc}
\hline Superficie (ha) & $<\mathbf{4}$ & $\mathbf{4 - 1 5}$ & $\mathbf{1 6 - 4 0}$ & $\mathbf{4 1 - 1 0 0}$ & $>\mathbf{1 0 0}$ & Total \\
\hline Número & 549 & 352 & 149 & 65 & 25 & $\mathbf{1 . 1 4 0}$ \\
Porcentaje & 48,15 & 30,87 & 13,07 & 5,70 & 2,21 & $\mathbf{1 0 0}$ \\
\hline
\end{tabular}

Fuente: Elaboración propia a partir de datos de EUROSTAT (2009).

Tabla 10

Explotaciones olivareras

\begin{tabular}{lccccc}
\hline $\begin{array}{l}\text { Contexto } \\
\text { Geográfico }\end{array}$ & Explotaciones & $\begin{array}{c}\text { Superficie } \\
\text { (ha) }\end{array}$ & $\begin{array}{c}\text { Olivos } \\
\text { (millones) }\end{array}$ & $\begin{array}{c}\text { Tamaño } \\
\text { medio (ha) }\end{array}$ & $\begin{array}{c}\text { Número medio } \\
\text { de olivos por } \\
\text { explotación }\end{array}$ \\
\hline Mundial & 2.600 .000 & 10.700 .000 & 1.400 & 4,1 & 538 \\
UE & 1.900 .000 & 5.874 .100 & 872 & 3,0 & 459 \\
España & 550.000 & 2.552 .700 & 319 & 4,5 & 580 \\
Andalucía & 320.000 & 1.515 .320 & 179 & 4,7 & 559 \\
\hline
\end{tabular}

Fuente: Elaboración propia a partir de MARM (2009).

guiente, a la mejora competitiva del modelo tradicional o extensivo. Entre estas se encuentran:

\section{Evolución, avance y adecuación en el modo de explotación}

Dentro de este apartado se incluyen todas las modificaciones estructurales que reducen los costes globales, adaptando la explotación en la mayor medida posible a los distintos sistemas de mecanización.

Entre tales modificaciones se encuentra el reducir el número de pies del olivo a 2 (evidentemente adecuando la masa vegetal de ambos) de este modo se optimiza la recolección mecánica (Vilar et al., 2009b), la instauración de un sistema de irrigación, siempre y cuando sea posible, que restará vecería en las sucesivas cosechas, etc.

El análisis económico de actividad para una explotación tradicional que ha seguido estas medidas (Tabla 11), pone de relieve que se produce un incremento en la renta neta o líquida del olivicultor del $55,11 \%$, más de mil euros en términos absolutos, de los cuales casi el $14 \%$ se deben a la reducción en los costes de recolección.

\section{Búsqueda de efecto concentración:} cultivo compartido

La explotación no profesionalizada motivada por el reducido tamaño, y la elevada dispersión de las explotaciones, como se ha descrito anteriormente, deja a los olivicultores de segunda actividad, dos alternativas en el escenario de desaparición de subvenciones: la venta de la explotación, o el desempeño de dicha actividad de manera profesionalizada, sin necesidad de adquisición de tierras adicionales recurriendo a la denominada explotación compartida o a la variante de explotación asistida o subcontratada.

En cuanto al cultivo o explotación compartida, éste consiste en aunar las distintas explotaciones propiedad de diferentes personas (no es necesario realizar una unificación patrimonial) y explotarlas con medios comunes repercutiendo positivamente en una reducción de costes.

En la Tabla 12 se realiza un análisis económico de actividad para cultivo tradicional explotado mediante cultivo compartido en una finca situada en el seno de un total de 12.900 olivos cuyas fincas se catalogan como de media dispersión; ésta en cuestión es de 1.800 olivos tradicionales a $10 \times 10$, de regadío y variedad picual. La concentración de olivar se debe a la actividad conjunta de 6 familiares, y cuya actividad principal, no es la explotación de olivar.

Si se analizan los datos, mediante dicho sistema de explotación, conseguimos un incremento de la renta neta o líquida del olivicultor del $21 \%$, con respecto al análisis inicial efectuado del cultivo tradicional, y del $4,8 \%$ si comparamos con el segundo análisis resultante de la evolución, básicamente dicha reducción se genera vía descenso en el coste de recursos humanos y disminución de costes de amortización o depreciación. Igualmente tales efectos positivos, se ven potenciados por el descenso de la dispersión que genera la concentración de explotaciones.

\section{Búsqueda de los efectos profesionalización e integración: explotación asistida}

Se trata de un sistema de cultivo, mediante el cual, una persona, sociedad u otra entidad, con los suficientes recursos humanos y mecánicos o con posibilidad de obtenerlos para la explotación de oli- 
Tabla 11

Análisis económico del cultivo tradicional con medidas de modernización

\begin{tabular}{lcc}
\hline Producción por hectárea & $\begin{array}{c}\text { Gastos } \\
\text { (euros) }\end{array}$ & $\begin{array}{c}\text { Ingresos } \\
\text { (euros) }\end{array}$ \\
\hline kg /olivo (68,00) & $3.928,36$ \\
Ingresos (Kg. 0,53 euros) & 963,56 \\
Subvención a la produc. (Kg. 0,13 euros) & 59,12 \\
Poda y eliminación de leña (Bianual) & 25,00 \\
Arijas (Picado, soplado de suelos, etc.) & 127,00 \\
Abonado / estercolado & 49,23 \\
Tratamientos (repilo, prays y mosca) & 12,90 \\
Despestugo / desvareto (químico) & 667,08 \\
Recolección & 19,02 & \\
Transporte de tajo a almazara & 0,09 \\
Extracción (euros / Kg.) & $2.012,35$ \\
Total & \multicolumn{2}{c}{$2.879,57$} \\
Renta Neta & \multicolumn{2}{c}{$36.060,72$} \\
Valor inmovilizado de mercado (euros) & \multicolumn{2}{c}{$7,98 \%$} \\
Margen neto de Explotación (\%)
\end{tabular}

*Explotación con medidas de reducción del número de pies de olivo a 2, instauración de sistemas de irrigación y mecanización de procesos como tratamiento fitosanitario, entre otros.

Fuente: Elaboración propia a partir de datos de la explotación la Jabonera de Polaino ubicada en Chilluévar (Jaén-España) (campaña 2007/08).

Tabla 12

Análisis económico de actividad para cultivo tradicional explotado mediante cultivo compartido

\begin{tabular}{|c|c|c|}
\hline Producción por hectárea & $\begin{array}{l}\text { Gastos } \\
\text { (euros) }\end{array}$ & $\begin{array}{c}\text { Ingresos } \\
\text { (euros) }\end{array}$ \\
\hline \multicolumn{3}{|l|}{$\mathrm{kg}$ /olivo $(65,12)$} \\
\hline Ingresos (Kg. 0,53 euros) & & $3.865,52$ \\
\hline Subvención a la produc. (Kg. 0,13 euros) & & 948,15 \\
\hline Poda y eliminación de leña (Bianual) & 49,12 & \\
\hline Arijas (arado, rastreado, etc.) & 38,43 & \\
\hline Abonado / estercolado & 28,17 & \\
\hline Tratamientos (repilo, prays y mosca) & 38,10 & \\
\hline Despestugo / desvareto & 9,19 & \\
\hline Recolección & 495,12 & \\
\hline Transporte de tajo a almazara & 9,32 & \\
\hline Extracción (euros / Kg.) & 0,09 & \\
\hline Total & $1.390,86$ & $4.813,67$ \\
\hline Renta Neta & \multicolumn{2}{|c|}{$3.423,81$} \\
\hline Valor inmovilizado de mercado (euros) & \multicolumn{2}{|c|}{$36.060,72$} \\
\hline Margen neto de Explotación (\%) & \multicolumn{2}{|c|}{$9,40 \%$} \\
\hline
\end{tabular}

Fuente: Elaboración propia a partir de datos de la explotación la Almedina, Término Municipal de Peal de Becerro (Jaén-España) (campaña 2007/08).

var, y que tiene como primera actividad el cultivo de olivar extensivo o tradicional, cuenta con recursos ociosos por no trabajar el suficiente número de explotaciones. Mediante, arrendamiento, aparcería o simple prestación de servicios elimina el efecto dispersión, y mediante efecto crecimiento alcanza el punto óptimo de rentabilidad.

En la Tabla 13 se recogen los datos procedentes de una explotación, La Fuentecilla con 1.100 olivos tradicionales con marco 10 x 10 de regadío y 
Tabla 13

Análisis económico de actividad para cultivo tradicional explotado mediante cultivo asistido o subcontratado

\begin{tabular}{|c|c|c|}
\hline Producción por HECTÁREA & $\begin{array}{l}\text { Gastos } \\
\text { (euros) }\end{array}$ & $\begin{array}{c}\text { Ingresos } \\
\text { (euros) }\end{array}$ \\
\hline \multicolumn{3}{|l|}{$\mathrm{kg}$ /olivo $(69,09)$} \\
\hline Ingresos (Kg. 0,53 euros) & & $4.174,41$ \\
\hline Subvención a la produc. (Kg. 0,13 euros) & & $1.023,91$ \\
\hline Poda y eliminación de leña (Bianual) & 37,02 & \\
\hline Arijas (arado, rastreado, etc.) & 29,41 & \\
\hline Abonado / estercolado & 19,13 & \\
\hline Tratamientos (repilo, prays y mosca) & 39,14 & \\
\hline Despestugo / desvareto & 8,21 & \\
\hline Recolección & 472,57 & \\
\hline Transporte de tajo a almazara & 8,01 & \\
\hline Extracción (euros / Kg.) & 0,09 & \\
\hline Total & $1.322,35$ & $5.198,32$ \\
\hline Renta Neta & \multicolumn{2}{|c|}{$3.875,97$} \\
\hline Valor inmovilizado de mercado (euros) & \multicolumn{2}{|c|}{$36.060,72$} \\
\hline Margen neto de Explotación (\%) & \multicolumn{2}{|c|}{$10,70 \%$} \\
\hline
\end{tabular}

variedad picual. Dicha finca se encuentra explotada de manera subcontratada por un olivicultor, que desempeña dicha actividad, en primer término, y que explota un total de 13.000 olivos tanto colindantes, como con cierto índice de dispersión.

Analizando los datos, se observa una mejora del $24 \%$ de la renta neta del olivicultor, si se compara con el análisis efectuado para la hectárea de olivar tradicional, una vez aplicados ciertos elementos de evolución o adecuación, la variación positiva de dicho resultado líquido es del 6,8\%, todo ello generado vía reducción de costes en recursos humanos y de amortización o depreciación, y potenciado por los efectos crecimiento, concentración o no dispersión, etc.

Evidentemente todos estos efectos, como se ha podido observar, son combinables y acumulables, entre si, generando ahorros en los costes de recolección, de acuerdo con los ensayos realizados, de hasta $48 \%$, a la vez que la incidencia sobre la renta neta del olivicultor se ve positivamente afectada por incrementos que alcanzan el $23 \%$.

No obstante cuanto mayor es el efecto crecimiento y concentración conseguido, vía descenso de la dispersión entre las distintas explotaciones, superiores serán el efecto disminución sobre los costes de recolección, y la incidencia positiva sobre el nivel de renta neta del olivicultor tradicional.

\section{CONCLUSIONES}

Como colofón al presente trabajo a continuación se enuncian las siguientes conclusiones o reflexiones finales:
En la actualidad se consumen en el mundo más de 140 millones de t de grasas de origen vegetal o animal, el aceite de oliva supone el 2,07\% del total.

Existen cuatro modos distintos de explotación del olivo: cultivo marginal, tradicional, intensivo y superintensivo, siendo los dos primeros los que inferior rendimiento obtienen de dicha explotación.

Cinco son los elementos básicos y estratégicos con los que incidir en el modo de explotación tradicional o extensivo para asegurar su continuidad, consolidación, y nivel de rentabilidad: la concentración de oferta, la promoción, la calidad, la seguridad agroalimentaria y la competitividad vía liderazgo en costes.

La adecuación, evolución y avance aplicado de manera eficaz en el modo de explotación tradicional garantiza elevada reducción de costes, con el consiguiente incremento de rentabilidad.

Mediante las estrategias de explotación denominada cultivo compartido y cultivo asistido se reducen la dispersión a la vez que se eleva el nivel de competitividad del modo de explotación extensiva o tradicional, garantizando y consolidando adecuados niveles de renta neta para el olivicultor fundamentalmente vía efecto concentración y consiguiente reducción de costes.

\section{REFERENCIAS}

Abos J, Fabo JM, Agreda J, Otazu J. 2007. Nuevas plantaciones de olivo en regadío. Rentabilidad y futuro. Navarra Agraria no noviembre-diciembre, 29-33. 
Alba J. 2009. Estado actual de la tecnología de elaboración de aceite de oliva virgen. II Jornadas Nacionales del Grupo de Olivicultura de la Sociedad Española de Ciencias Hortícolas (SECH). Tarragona, marzo, 48-49.

Arriaza M, Barea F, Ruiz P, Lucena B. 2002. Reforma de la OCM del aceite de oliva: hacia un sistema desacoplado. Informe anual del sector agrario de Andalucía, Unicaja, Málaga.

Asociación Argentina de Grasas y Aceites (ASAGA). 2009. Resumen anual de magnitudes. Publicación trimestral ASAGA 73, 612-690.

Asociación Española De Municipios Del Olivo (AEMO). 2003. AEMO, al lado del olivar de montaña y de baja producción. Mercacei 33, 136-140.

Atance I, Bardaji I, Tió C. 2001. Fundamentos económicos de la multifuncionalidad agraria e intervención pública. IV Congreso Hispano-Portugués de Estudios Rurales. Santiago de Compostela, junio.

Atance I, Tió C. 2000. La multifuncionalidad en la agricultura: aspectos económicos e implicaciones sobre la política agraria. Revista de estudios Agro-Sociales $189,29-48$.

Barranco D. 2007a. Modelos productivos en el olivar tradicional, intensivo y superintensivo". Comunicaciones. Jornadas sobre el Futuro del Olivar y del Aceite de Oliva en Andalucía. Jaén, noviembre.

Barranco D. 2007b. Variedades adaptadas al olivar superintensivo. Jornadas técnicas el futuro de la olivicultura con suficiencia de recursos. Olivar intensivo versus superintensivo. Córdoba, marzo.

Canals J. 1991. Competitividad internacional y estrategia de la empresa. Ariel Economía, Barcelona.

Consejo Oleícola Internacional (COI). 2000. Políticas oleícolas nacionales. Consejo Oleícola Internacional, Madrid.

Consejo Oleícola Internacional (COI). 2009. Producción, importación, exportación y consumo mundial de aceite de oliva 1997-2008/09. Disponible en: www.internationaloliveoil.org/

EUROSTAT. 2009. Agricultural statistics. Main Result. Disponible en: http://epp.eurostat.ec.europa.eu/portal/ page/portal/agriculture/introduction

García E. 2001. Los objetivos de la sociedad cooperativa almazarera. CIRIEC-España 37, 203-221.

Hidalgo JC, Vega V, Fereres E, Pastor M. 2006. Viabilidad económica de plantaciones superintensivas en Andalucía. Vida Rural 238, 60-66.

Humanes J, Pastor M, Vega V, Castro J. 1998. Diseño y manejo de plantaciones de oliva. Junta de Andalucía. Consejería de Agricultura y Pesca, Sevilla.

Lamo De Espinosa P. 2008. La defensa de la competencia en la legislación de la Unión Europea y en España, e investigación sobre las resoluciones dictadas por el Tribunal de defensa de la Competencia en el sector agroalimentario. Tesis Doctoral. Escuela Técnica Superior de Ingenieros Agrónomos de Madrid, Madrid.

León L, De La Rosa R, Guerrero N, Rallo L, Barranco D, Tous J, Romero A, Hermoso JF. 2006. Ensayos de variedades de olivo en plantaciones de alta densidad. Comparación de resultados entre Andalucía y Cataluña. Fruticultura Profesional (Especial Olivicultura IV) $160,21-26$.

Leone FG. 2000. Globalización del mercado del aceite de oliva y competitividad del sector en Italia. Una comparación internacional. Olivae 83, 11-14.

Massot A. 2005. La reforma de la PAC de 2002/2003 y sus corolarios: la agricultura europea ante un nuevo escenario, en Martín JM. PAC: claves para Andalucía, 2004-2005. ETEA, Córdoba, 65-80.

Ministerio De Medio Ambiente Y Medio Rural Y Marino (MARM). 2009. Encuesta de superficies y rendimientos de cultivos y Avances de superficies y producciones de cultivos. Disponible en http://www.mapa.es/ es/estadística.

Mulero A. 2003. Protección y gran propiedad en Sierra Morena: el parque natural de la Sierra de Hornachuelos (Córdoba) como caso emblemático. Papeles de Geografía 38, 115-136.

Navarro C, Parra MA. 1997. Plantación, en Barranco D, Fernández-Escobar R, Rallo L. El cultivo del olivo. Junta de Andalucía y Mundi-Prensa, Madrid, 170-189.

Pastor M, Vega V, Hidalgo JC. 2005. Ensayos en plantaciones de olivar superintensivas e intensivas. Vida Rural 218, 30-40.

Pastor M, Vega V, Hidalgo J, Fereres EE, Hidalgo J. 2007. Viabilidad agronómica y económica de las plantaciones superintensivas en Andalucía. ASAJAJaén 312, 22-32.

Porter M E. 2003. Ventaja competitiva. Creación y sostenimiento de un desempeño superior. Compañía Editorial Continental, México

Puentes R, Velasco MM. 2009. Importancia de las sociedades cooperativas como medio para contribuir al desarrollo económico, social y medioambiental, de forma sostenible y responsable. Revista de Estudios Cooperativos (REVESCO) 99, 104-129.

Reig E. 2002. La multifuncionalidad del mundo rural. In formación Comercial Española 803, 33-44.

Reig E. 2007. Fundamentos económicos de la multifuncionalidad, en Gómez-Limón JA, Barreiro J. (Coords.). La multifuncionalidad de la agricultura en España. Conceptos, aspectos horizontales, cuantificación y casos prácticos. EUMEDIA S.A. y Ministerio de Agricultura, Pesca y Alimentación, Madrid, 13-39.

Tamames R, Rueda A. 2005. Estructura económica de España. Alianza Editorial, Madrid.

Tió C. 2007. La reforma de la PAC y sus impacto en el sector olivarero, en Sillero J. I Congreso de la Cultura del aceite, Instituto de Estudios Jiennenses, Jaén, 323-340.

Tous J, Romero A, Hermoso JF, Mallén N. 2007. Sistemas de producción en seto. Experiencias en Cataluña. Agricultura Revista Agropecuaria 896, 360-367.

Vilar J. 2006. Análisis económico internacional del proceso de elaboración de aceite de oliva. Retos y estrategias de futuro. Westfalia Separator Andalucía, S. L, Úbeda (Jaén).

Vilar J, Moya M, Espínola F. 2005. Orígenes, evolución y actual tendencia del sector oleícola-olivarero. I Congreso de Cultura del Olivo, Jaén, octubre, 441-455.

Vilar J, Velasco MM. 2007. Influencia en el precio del aceite de oliva de la utilización de otros aceites vegetales para la elaboración de biocombustibles. Seminario Estatal del sector del olivar de COAG, Zaragoza, octubre.

Vilar J, Velasco MM, Puentes R. 2009a. Efectos de la implantación de un sistema de gestión de la calidad en el proceso de elaboración de aceite de oliva. Un estudio internacional. Agroalimentaria 29, 544-551.

Vilar J, Velasco MM, Puentes R. 2009b. El olivar tradicional giennense. Estrategias de futuro para el posible contexto de ausencia de ayudas. Fundación Caja Rural de Jaén, Jaén. 